\title{
RESEARCH
}

\section{THE EFFECT OF COVID-19 PANDEMIC ON SARCOPENIA, QUALITY OF LIFE AND PAIN: A ONE-YEAR FOLLOW-UP STUDY}

Turkish Journal of Geriatrics

DOI: 10.31086/tigeri.2021.230

2021; 24(3): 330-343

- Fulya BAKILAN

- Merih ÖZGEN ${ }^{2}$

- Burcu ORTANCA ${ }^{1}$.

- Onur ARMAĞAN ${ }^{2}$

- Ayşe EKIM ${ }^{1}$

- Funda Taşçıoğlu BERKAN²

- Fezan Şahin MUTLU3

CORRESPONDANCE

${ }^{1}$ Fulya BAKILAN

Eskişehir City Hospital, Physical Medicine and

Rehabilitation, Eskişehir, Turkey

Phone: +905057737335

e-mail: fulyabakilan@gmail.com

Received: Jul 14, 2021

Accepted: Aug 30, 2021

${ }^{1}$ Eskişehir City Hospital, Physical Medicine and Rehabilitation, Eskişehir, Turkey

2 Eskişehir Osmangazi University, Department of Physical Medicine and Rehabilitation, Eskişehir, Turkey

${ }^{3}$ Eskişehir Osmangazi University, Department of Biostatistics, Eskişehir, Turkey

\section{Abstract}

Introduction: This study evaluated the effect of coronavirus disease 2019 pandemic on sarcopenia, quality of life and pain severity in elderly patients and investigated which patients were affected more from this pandemic (sarcopenic or not, males or females, and physically active or inactive).

Materials and Methods: A total of 225 elderly patients with SARC-F, visual analogue scale-musculoskeletal pain scores, handgrip strength, chair stand test, short form-36, demographic data and phone number records in July 2020 were included in this study. Follow-up was conducted in June 2021 with telephonic interviews. During follow-up, SARC-F, 10-point Likert pain scale, Short Form-36 and physical activity were evaluated.

Results: After one year of the COVID-19 pandemic, almost all short form36 scores decreased; however, the pain and SARC-F scores did not show a significant change. Decrease in short form-36 scores were higher in patients without sarcopenia than in those with sarcopenia. Decrease in Short Form36 scores were higher in women than in men. Increase in pain scores (active: $p=0.851$, inactive: $p=0.005$ ) and decrease in Short Form-36 scores, were higher in physically active patients than in physically inactive patients. SARC-F scores did not change in any of the study groups.

Conclusion: After one-year follow-up in elderly patients, quality of life decreased, severity of pain increased in physically inactive, and SARC-F was unaffected. Females, non-sarcopenic, and physically inactive patients were found to be more affected by the COVID-19 pandemic.

Keywords: COVID-19; Pain; Quality of life; Sarcopenia. 


\section{INTRODUCTION}

In December 2019, Wuhan city in China reported the first confirmed case of coronavirus disease 2019 (COVID-19), which subsequently caused worldwide outbreak. Consequently, a pandemic was declared by the World Health Organization (WHO). While it is important to focus on controlling COVID-19, it is also necessary to identify the long-term effects of the pandemic. COVID-19 has been shown to have substantial long-term impact on several organs of the musculoskeletal system, especially in the geriatric population, because of restrictions and social isolation. One of the consequences of the COVID-19 pandemic has been a decrease in physical activity (1), which is a risk factor for pain (2), reduced quality of life (3), and sarcopenia (4). In addition, social isolation and increased loneliness (5) can lead to anxiety and depression resulting in lower quality of life in the elderly (6). Furthermore, decreased sunlight exposure, which is associated with lower vitamin D concentrations, is a risk factor for pain and sarcopenia (4).

According to the European Working Group on Sarcopenia in Older People (EWGSOP2), the SARC-F scale is recommended to identify cases of sarcopenia. If the results of SARC-F are negative, the patients are considered non-sarcopenic. In clinical practice, after a positive SARC-F score, low muscle strength is sufficient to start intervention (7).

Only a few studies have investigated the effects of COVID-19 pandemic on the musculoskeletal system $(8,9)$. One of the studies reported increased sarcopenia rates in geriatric patients with diabetes (8). Another study reported pain augmentation in adults during the pandemic (9). Considering that the musculoskeletal system can be affected by many conditions, such as physical activity, sarcopenia, and gender, evaluations should be made taking these conditions into account.

The aims of this one-year follow-up cohort study were as follows: 1) to evaluate the effect of the COVID-19 pandemic on sarcopenia using the SARC-F scale, quality of life, and pain severity in geriatric patients; and 2) to investigate which patients were affected more when classified on the basis of the presence or absence of sarcopenia, sex, and being physically active or inactive.

\section{MATERIALS AND METHODS}

This cohort study was conducted in patients aged 60 years or older who were admitted to a physical medicine and rehabilitation outpatient clinic in July 2020. Patients with SARC-F scores, visual analog scale (VAS)-musculoskeletal pain scores, handgrip strength, chair stand test, short form-36 (SF-36), demographic data, and phone number records in July 2020 were included in this study. Follow-up was conducted via telephone interviews in June 2021. Patients with an additional chronic disease or surgery within one year; active cancer, cerebrovascular stroke, and other neurological disorders, acute/ subacute pain, active infection, active arthritis, impaired cognitive status, advanced hearing loss, those who ever had COVID-19 infection or underwent treatment in a hospital within the past one year and immobilised patients were excluded from the study.

In July 2020, 225 patients were evaluated in terms of demographic data (age; sex; weight; height; education/working/family status; presence of concurrent diseases, such as hypertension, diabetes mellitus, chronic obstructive pulmonary disease, thyroid problems; cane usage; smoking), VAS pain, SARC-F score, handgrip strength, chair stand test, and SF-36 by a physical medicine and rehabilitation specialist (face to face) in physical medicine and rehabilitation outpatient clinic. In June 2021, another physical medicine and rehabilitation specialist who was blind to 2020 records conducted telephone interviews with those patients. Any disease, such as cancer, infection, COVID-19 infection and surgery within one year; the rate of musculoskeletal pain with 10-point likert scale; SARC-F; SF-36; and regular exercise were recorded via telephone interviews 
with patients themselves (10-15 minutes), without any visual interview. Telephone interviews were held those who were available at that time, appointments were made those who were not available.

The VAS was used to measure general body musculoskeletal pain, which was assessed from 0 (no pain) to 10 (worst possible pain) (10). One year later, 10-point likert was used for general body musculoskeletal pain. The question for this measurement was the following: "How would you rate your general body pain on a 0-10 scale at the present time? Zero is no pain and ten is pain as bad as could be."

Quality of life assessment was performed using the SF-36 with 36 items on physical function, physical role, emotional role, energy, body pain, mental health, general health, and social function. Each scale ranges from 0 (poor health) to 100 (perfect health) (11). Patients spent almostly an average of 5-10 minutes on answering the SF-36 by telephone.

Ambulation, strength, history of falls, rising from a chair, and stair climbing were evaluated using the SARC-F scale which assesses the risk of sarcopenia. A score lower than 4 was defined as non-sarcopenic. The chair stand test measures the time taken for five rounds of rising from the sitting position without using arms, and was defined as low when the time taken was $>15 \mathrm{~s}$. Grip strength was measured with a hand-held dynamometer (Baseline, White Plains, New York, USA), and the cut-off thresholds were 32 $\mathrm{kg}$ for men and $22 \mathrm{~kg}$ for women). The chair stand test and grip strength were measured only in July 2020. Probable sarcopenia was defined according to the EWGSOP2 algorithm as having a low SARC-F score and low muscle strength (grip strength/chair stand test) (7).

Physical activity was assessed by asking patients to perform vigorous and moderate physical activity. Vigorous physical activities were defined as physical effort resulting in faster breathing than normal. Moderate physical activities were defined as moderate physical effort resulting in somewhat faster breathing than normal. Patients were asked the following questions: 1) 'How many minutes do you spend on vigorous physical activity on an average day for one year?'; 2) 'How many minutes do you spend on moderate physical activity on an average day for one year?'. According to the WHO guidelines (12), at least $150 \mathrm{~min}$ of moderate and/or 75 min of vigorous physical activity per week for adults and elderly was defined as 'regular physical activity'.

Verbal informed consent was provided by the patients before the telephonic interview. Ethics approval was received from the local ethics committee 30/03/21-09 (25403353-050.99-181555).

\section{Statistical Analysis}

The distribution of each continuous variable was tested for normality using the Shapiro-Wilk test and is expressed as median value (\%25-\%75). Non-normally distributed variables were performed using the Mann Whitney $U$ and Wilcoxon Signed Ranks Test for continuous variables. The categorical variables are expressed in frequencies and percentages. The Chi-square test was used to compare categorical variables. A p-value $<0.05$ was considered significant. All analyses were performed using the SPSS version 22.0 software (SPSS Inc., Chicago, IL, USA).

\section{RESULTS}

One patient died during the study period. Twenty (8.8\%) patients with COVID-19 infection, six (2.6\%) with an additional chronic disease (such as cancer, vision loss, cardiac disease, bone marrow disease), three (1.3\%) who underwent surgery (discectomy, prostate), four (1.7\%) with acute pain in the last month, and 30 (13.3\%) who were not contacted by telephone were excluded. Finally, 161 patients (125 women, 36 men; mean age, $68.4 \pm 6.2$ years) who met the inclusion criteria were enrolled in our study. The demographic characteristics of the patients are presented in Table 1. 
Table 1. Demographic characteristics of patients

\begin{tabular}{|l|c|}
\hline & Frequencies \\
$\mathbf{n}(\%)$
\end{tabular}

( $k g=$ kilograms, $B M I=$ Body mass index)

Comparison of patients between June 2020 and July 2021 showed that after approximately one year of COVID-19 pandemic, their SF-36 scores decreased except 'role emotional' (physical function: $p<0.001$, role physical: $p=0.007$, bodily pain: $p=0.011$, energy: $p<0.001$, mental health: $p<0.001$, general health: $p=0.006$, social function: $p=0.001$ ) However, pain scores and SARC-F scores did not show a statistically significant change (Table 2).

Comparison of patients with and without sarcopenia showed that patients with sarcopenia had higher SARC-F and pain scores and lower SF36 scores in both 2020 and 2021. In patients with probable sarcopenia, physical function and general health decreased after one year of COVID-19 pandemic. In patients without sarcopenia, only SARC-F and pain scores and emotional role did not change; however, other parameters changed after one year of COVID-19 pandemic (physical function, $\mathrm{p}<0.001$; physical role, $p=0.024$; bodily pain, $p=0.019$; energy, $p<0.001$; mental health, $p<0.001$; general health, $p=0.031$; social function, $p=0.004)$. On comparing patients with probable sarcopenia with those without sarcopenia, age $(p=0.006)$ was found to be lower and the rate of regular exercise $(p=0.017)$ was higher in the no sarcopenia group (Table 3).

For female patients, most of the SF-36 scores (physical function, $<0.001$; physical role, $\mathrm{p}=0.019$; energy, $p=0.002$; mental health, $p<0.001$; general health, $p=0.014$; social function, $p=0.004$ ) decreased within one year of the pandemic. For male patients, three of the SF-36 parameters decreased (physical function, $p=0.004$; energy, $p=0.029$; mental health, $p=0.028$ ). Comparison of patients according to sex showed that females were found to have significantly higher SARC-F and pain scores, whereas many of the SF-36 scores (physical function, physical role, emotional role, energy, mental health) were lower than those of males in both 2020 and 2021. However, social function was similar between the two groups in both 2020 and 2021. On the other hand, females were found to have lower bodily pain and 
Table 2. Comparison of SARC-F, pain and SF-36 scores of patients between July 2020 (before) and June 2021 (after)

\begin{tabular}{|l|c|c|c|}
\hline \multicolumn{1}{|c|}{ Variables } & $\begin{array}{c}\text { Before } \\
\text { (median 25-75\%) }\end{array}$ & $\begin{array}{c}\text { After } \\
\text { (median 25-75\%) }\end{array}$ & p \\
\hline SARC-F score & $3(1-5)$ & $3(1-5)$ & 0.452 \\
\hline Pain scores & $6(2-8)$ & $5(3-8)$ & 0.066 \\
\hline SF-36 subgroups & & $55(35-75)$ & $<0.001$ \\
\hline Physical function & $60(35-80)$ & $50(0-100)$ & 0.007 \\
\hline Role physical & $50(0-100)$ & $33.3(0-66.7)$ & 0.208 \\
\hline Role emotional & $33.3(0-66.1)$ & $55(32-77)$ & 0.011 \\
\hline Bodily pain & $55(32-88)$ & $42.5(25-60)$ & $<0.001$ \\
\hline Energy & $45(27-60)$ & $60(40-76)$ & $<0.001$ \\
\hline Mental health & $60(44-76)$ & $60(45-70)$ & 0.006 \\
\hline General health & $60(45-70)$ & $100(50-100)$ & 0.001 \\
\hline Social function & $100(50-100)$ & & \\
\hline
\end{tabular}

general health than males in 2020; after one year, these values were found to be significantly similar between females and males (Table 4).

Male patients had regular physical activity compared to female patients $(p=0.003)$. Many of the parameters did not change during the one-year pandemic in patients who had regular physical activity; only mental health $(p=0.034)$ and energy $(p=0.025)$ decreased. However, in patients who did not engage in regular physical activity, many of the parameters changed within one year; only SARC-F scores and emotional role did not change. Their pain $(p=0.005)$ scores increased, and all of the SF36 parameters except emotional role (physical function: $p<0.001$, role physical: $p=0.002$, bodily pain: $p=0.021$, energy: $p=0.003$, mental health: $p<0.001$, general health: $p<0.001$, social function: $p=0.007$ ) decreased. Comparison of patients according to physical activity showed that the patients who regularly engaged in physical activity had lower SARC-F scores and higher SF-36 scores in both 2020 and 2021. Furthermore, pain $(p=0.001)$ scores increased significantly more in patients who did not engage in regular physical activity than in those who performed regular physical activity after one year of the pandemic (Table 5).

\section{DISCUSSION}

Restrictions, travel bans, social isolation, and fear of infection during the COVID-19 pandemic have resulted in an extended home stay for the geriatric population. This study aimed to investigate the effect of the COVID-19 pandemic on the severity of pain, quality of life, and sarcopenia in geriatric patients. Therefore, other causes, such as cancer, infection, and surgery, which may also affect these situations within the past one year, were excluded from our study. Patients who were evaluated faceto-face for pain, sarcopenia, and quality of life in July 2020, were followed up with telephonic interviews in June 2021 as telehealth care became widely used because of the COVID-19 pandemic (13). Within one year, almost all quality-of-life parameters decreased; however, the severity of musculoskeletal pain and SARC-F scores did not change. The severity of pain, quality of life, and SARC-F scores were 
Table 3. Comparison of age, physical activity levels, SARC-F, pain and SF-36 scores of both sarcopenia probable and no sarcopenia patients between July 2020 (before) and June 2021 (after)

\begin{tabular}{|c|c|c|c|c|}
\hline Variables & & $\begin{array}{c}\text { Sarcopenia probable } \\
\text { (median 25-75\%) } \\
(n=35)\end{array}$ & $\begin{array}{c}\text { No sarcopenia } \\
\text { (median 25-75\%) } \\
(n=126)\end{array}$ & $p$ \\
\hline Age (years) & & $70(65-78)$ & $67(61.7-70)$ & 0.006 \\
\hline Gender(female/male)n (\%) & & $31(89 \%) / 4(11 \%)$ & $94(75 \%) / 32(25 \%)$ & 0.058 \\
\hline Regular physical activity $\mathrm{n}(\%)$ & & $7(20 \%)$ & $53(42 \%)$ & 0.017 \\
\hline \multirow{3}{*}{ SARC-F Score } & Before & $5(4-8)$ & $2.5(1-4)$ & $<0.001$ \\
\hline & After & $6(4-7.25)$ & $3(1-4)$ & $<0.001$ \\
\hline & $\mathrm{p}$ & 0.545 & 0.638 & \\
\hline \multirow[t]{3}{*}{ Pain scores } & Before & $8(6-8)$ & $5(0-7)$ & $<0.001$ \\
\hline & After & $8(5-9)$ & $5(2-8)$ & $<0.001$ \\
\hline & $\mathrm{p}$ & 0.578 & 0.083 & \\
\hline \multicolumn{5}{|l|}{ SF-36 subgroups } \\
\hline \multirow[t]{3}{*}{ Physical function } & Before & $30(20-50)$ & $70(50-85)$ & $<0.001$ \\
\hline & After & $25(15-40)$ & $65(45-80)$ & $<0.001$ \\
\hline & $\mathrm{p}$ & 0.008 & $<0.001$ & \\
\hline \multirow[t]{3}{*}{ Physical role } & Before & $0(0-50)$ & $75(25-100)$ & $<0.001$ \\
\hline & After & $0(0-50)$ & $75(25-100)$ & $<0.001$ \\
\hline & $\mathrm{p}$ & 0.131 & 0.024 & \\
\hline \multirow[t]{3}{*}{ Emotional role } & Before & $0(0-33.3)$ & $66.7(33.3-100)$ & $<0.001$ \\
\hline & After & $0(0-33.3)$ & $66.7(33-100)$ & $<0.001$ \\
\hline & $\mathrm{p}$ & 0.414 & 0.317 & \\
\hline \multirow[t]{3}{*}{ Bodily pain } & Before & $32.5(22.5-55)$ & $67(45-90)$ & $<0.001$ \\
\hline & After & $32.5(10-55)$ & $67(45-78)$ & $<0.001$ \\
\hline & $p$ & 0.218 & 0.019 & \\
\hline \multirow[t]{3}{*}{ Energy } & Before & $25(20-40)$ & $50(35-65)$ & $<0.001$ \\
\hline & After & $27.5(15-40)$ & $45(30-65)$ & $<0.001$ \\
\hline & $p$ & 0.333 & $<0.001$ & \\
\hline \multirow[t]{3}{*}{ Mental health } & Before & $44(32-60)$ & $68(52-80)$ & $<0.001$ \\
\hline & After & $46(36-57)$ & $64(44-76)$ & $<0.001$ \\
\hline & $\mathrm{p}$ & 0.142 & $<0.001$ & \\
\hline \multirow[t]{3}{*}{ General health } & Before & $40(30-55)$ & $65(50-70)$ & $<0.001$ \\
\hline & After & $40(28.7-60)$ & $60(50-70)$ & $<0.001$ \\
\hline & $p$ & 0.031 & 0.031 & \\
\hline \multirow[t]{3}{*}{ Social function } & Before & $62.5(25-100)$ & $100(59-100)$ & 0.005 \\
\hline & After & $62.5(34-100)$ & $100(50-100)$ & 0.013 \\
\hline & $p$ & 0.157 & 0.004 & \\
\hline
\end{tabular}

(SF-36: Short form-36) 
Table 4. Comparison of age, SARC-F, pain and SF-36 scores of patients between July 2020 (before) and June 2021 (after) according to gender

\begin{tabular}{|c|c|c|c|c|}
\hline Variables & & $\begin{array}{c}\text { Female } \\
\text { (median 25-75\%) } \\
(n=125)\end{array}$ & $\begin{array}{c}\text { Male } \\
\text { (median } 25-75 \%)(n=36)\end{array}$ & $p$ \\
\hline Regular physical activity (\%) & & $39(31.2 \%)$ & $21(58.3 \%)$ & 0.003 \\
\hline Age (years) & & $67(64-71)$ & $67.5(62.5-73)$ & 0.722 \\
\hline \multirow[t]{3}{*}{ SARC-F Score } & Before & $4(2-5)$ & $1.5(1-3.7)$ & 0.001 \\
\hline & After & $4(2-5)$ & $2(1-4)$ & 0.004 \\
\hline & $\mathrm{P}$ & 0.725 & 0.325 & \\
\hline \multirow[t]{3}{*}{ Pain scores } & Before & $6(3-8)$ & $4(0-6.2)$ & 0.008 \\
\hline & After & $5(3.5-8)$ & $5(0.5-7.7)$ & 0.037 \\
\hline & $\mathrm{P}$ & 0.240 & 0.086 & \\
\hline \multicolumn{5}{|l|}{ SF-36 subgroups } \\
\hline \multirow[t]{3}{*}{ Physical function } & Before & $55(30-72)$ & $80(65-95)$ & $<0.001$ \\
\hline & After & $50(30-70)$ & $75(55-90)$ & $<0.001$ \\
\hline & $\mathrm{p}$ & $<0.001$ & 0.004 & \\
\hline \multirow[t]{3}{*}{ Physical role } & Before & $50(0-100)$ & $100(56-100)$ & 0.001 \\
\hline & After & $50(0-100)$ & $87.5(50-100)$ & 0.002 \\
\hline & $\mathrm{P}$ & 0.019 & 0.165 & \\
\hline \multirow[t]{3}{*}{ Emotional role } & Before & $33.3(0-66.7)$ & $66.7(8-100)$ & 0.016 \\
\hline & After & $33.3(0-66.7)$ & $66.7(8-100)$ & 0.006 \\
\hline & $\mathrm{p}$ & 0.193 & 1.000 & \\
\hline \multirow[t]{3}{*}{ Bodily pain } & Before & 55 (32.5-77.5) & $67(55-90)$ & 0.006 \\
\hline & After & $55(32-77)$ & $62(45-90)$ & 0.083 \\
\hline & $\mathrm{P}$ & 0.061 & 0.054 & \\
\hline \multirow[t]{3}{*}{ Energy } & Before & $40(25-55)$ & $57(41-75)$ & $<0.001$ \\
\hline & After & $40(21-55)$ & $55(45-72)$ & $<0.001$ \\
\hline & $p$ & 0.002 & 0.029 & \\
\hline \multirow[t]{3}{*}{ Mental health } & Before & $60(40-74)$ & $76(56-80)$ & 0.005 \\
\hline & After & $56(40-71)$ & $72(56-76)$ & 0.005 \\
\hline & $\mathrm{p}$ & $<0.001$ & 0.028 & \\
\hline \multirow[t]{3}{*}{ General health } & Before & $60(40-70)$ & $62(55-75)$ & 0.037 \\
\hline & After & $60(40-70)$ & $60(50-73)$ & 0.156 \\
\hline & $\mathrm{p}$ & 0.014 & 0.210 & \\
\hline \multirow[t]{3}{*}{ Social function } & Before & $100(50-100)$ & $100(78-100)$ & 0.129 \\
\hline & After & $87.5(50-100)$ & $100(53-100)$ & 0.187 \\
\hline & $\mathrm{P}$ & 0.004 & 0.144 & \\
\hline
\end{tabular}

(SF-36: Short form-36) 
Table 5. Comparison of age, SARC-F, pain and SF-36 scores of patients between July 2020 (before) and June 2021 (after) according to making regular physical activity or not.

\begin{tabular}{|c|c|c|c|c|}
\hline Variables & & $\begin{array}{l}\text { Regular physical activity } \\
\text { (median } 25-75 \%)(n=60)\end{array}$ & $\begin{array}{l}\text { No regular physical activity } \\
\text { (median } 25-75 \%)(n=101)\end{array}$ & $p$ \\
\hline Age (years) & & $67.5(62.2-71)$ & $67(64-71)$ & 0.564 \\
\hline Gender (female/male) (n) & & $39 / 21$ & $86 / 15$ & 0.003 \\
\hline \multirow[t]{3}{*}{ SARC-F Score } & Before & $2(1-4)$ & $4(2-5)$ & 0.003 \\
\hline & After & $2(1-4)$ & $4(2-6)$ & 0.001 \\
\hline & $\mathrm{p}$ & 0.761 & 0.259 & \\
\hline \multirow[t]{3}{*}{ Pain scores } & Before & $5(0-8)$ & $6(4-8)$ & 0.059 \\
\hline & After & $4(2-6)$ & $6(4-8)$ & 0.001 \\
\hline & $\mathrm{p}$ & 0.851 & 0.005 & \\
\hline \multicolumn{5}{|l|}{ SF-36 subgroups } \\
\hline \multirow[t]{3}{*}{ Physical function } & Before & $72.5(50-85)$ & $55(30-70)$ & 0.001 \\
\hline & After & $70(45-85)$ & $50(30-70)$ & $<0.001$ \\
\hline & $p$ & 0.068 & $<0.001$ & \\
\hline \multirow[t]{3}{*}{ Physical role } & Before & $100(25-100)$ & $50(0-100)$ & 0.008 \\
\hline & After & $75(25-100)$ & $50(0-75)$ & 0.001 \\
\hline & $\mathrm{p}$ & 0.668 & 0.002 & \\
\hline \multirow[t]{3}{*}{ Emotional role } & Before & $66.7(0-100)$ & $33.3(0-66.7)$ & 0.011 \\
\hline & After & $66.7(33-100)$ & $33.3(0-66.7)$ & 0.001 \\
\hline & $\mathrm{p}$ & 0.752 & 0.051 & \\
\hline \multirow[t]{3}{*}{ Bodily pain } & Before & $67.5(45-90)$ & $55(32.5-77.5)$ & 0.057 \\
\hline & After & $67.5(45-80)$ & $55(32-77)$ & 0.061 \\
\hline & $\mathrm{p}$ & 0.247 & 0.021 & \\
\hline \multirow[t]{3}{*}{ Energy } & Before & $50(36.2-70)$ & $40(25-55)$ & $<0.001$ \\
\hline & After & $50(36-70)$ & $35(20-55)$ & $<0.001$ \\
\hline & $\mathrm{p}$ & 0.025 & 0.003 & \\
\hline \multirow[t]{3}{*}{ Mental health } & Before & $68(44-80)$ & $60(44-76)$ & 0.137 \\
\hline & After & $64(44-76)$ & $56(40-72)$ & 0.128 \\
\hline & $p$ & 0.034 & $<0.001$ & \\
\hline \multirow[t]{3}{*}{ General health } & Before & $65(50-75)$ & $55(40-67)$ & 0.017 \\
\hline & After & $65(51-73)$ & 55 (40-65) & 0.002 \\
\hline & $p$ & 0.945 & $<0.001$ & \\
\hline \multirow[t]{3}{*}{ Social function } & Before & $100(78-100)$ & $87.5(50-100)$ & 0.013 \\
\hline & After & $100(62-100)$ & 75 (50-100) & 0.014 \\
\hline & $p$ & 0.102 & 0.007 & \\
\hline
\end{tabular}

(SF-36: Short form-36) 
compared between female and male patients, patients with and without sarcopenia, and physically active and inactive patients.

Decreased physical activity, protein intake, sun exposure, increased sitting-screen time, stress, anxiety, and meal frequency have long-term adverse effects on muscle loss (4). To understand sarcopenic change, only the SARC-F score was used during the telephonic interview, and we could not perform grip strength and chair stand test measurements. The SARC-F scores were found to be similar between 2020 and 2021 in all study groups. A recent study in Japan reported that after the onset of the COVID-19 pandemic skeletal, muscle mass index had decreased in 56 geriatric diabetes patients compared to that before the pandemic with high mean follow-up duration (between the start and the end of the evaluation were 34 months) (8). The SARC-F is an inexpensive and practical method for evaluating sarcopenia in geriatric patients, with a sensitivity of $21 \%$ and specificity of $90 \%$ (14). It may not be appropriate to use SARC-F alone without objective measurements to evaluate annual changes in the geriatric population. We believe that there would have been a difference in our results if grip strength and chair stand test could be performed or the follow-up period was more than a year.

To the best of our knowledge, this is the first study to evaluate the effects of the COVID-19 pandemic on pain, SARC-F, and quality of life by comparing patients with and without sarcopenia according to the EWGSOP2 algorithm. Interestingly, in patients with sarcopenia, only general health and physical function decreased during the pandemic. Although all parameters were better in patients without sarcopenia in both 2020 and 2021, the pandemic affected patients without sarcopenia more than those with sarcopenia. Almost all quality of life parameters decreased more than those of patients with sarcopenia within one year. Patients with sarcopenia already have impaired physical function, mobility, social isolation, and reduced quality of life (15). Impaired physical function and mobility result in reduced physical activity, as observed in our study. Therefore, their daily lives may not have been affected to a large extent because of the restrictions and social isolation during the pandemic.

This study did not investigate how physical activity levels change during the pandemic because previous studies have already reported worldwide reduction in levels of physical activity reduced during the COVID-19 pandemic (1). In Turkey, the rate of geriatric physical activity was previously reported to be 33\% (16) during the COVID-19 pandemic, similar to the rate observed in our study (38\%). These low rates are associated not only with the pandemic but also with the habits of Turkish people. Before the pandemic, Akturk et al. (17) reported the rate of physical activity in geriatric population to be $32 \%$, which supports our findings. According to the $\mathrm{WHO}$, at least 150 min of moderate-intensity physical activity or 75 min of vigorous-intensity physical activity per week is recommended (12). Investigating the factors related to the recommended physical activity level showed that only energy and mental health were affected by the pandemic in patients who engaged in regular physical activity. On the other hand, musculoskeletal pain severity increased, and almost all quality-of-life parameters decreased in physically inactive patients. In addition, SARC-F scores were lower in the physically active patients than in the inactive patients in both 2020 and 2021. This result showed that physical inactivity is related to increased pain, sarcopenia, and decreased quality of life, and it is important for the elderly population to gain and continue physical activity behaviours during a pandemic. In elderly individuals, walking programs have been shown to positively impact the quality of life (18). Web-based home exercise programs or outdoor walking programs should be implemented, which can be useful for both increasing motivation and physical activity.

The relationship between quality of life and physical activity in older people has been reported in a 
systematic review (3). Physically inactive elderly individuals are at an increased risk of cardiovascular diseases, cancer, falls, disability, functional limitation, cognitive decline, and depression. Consequently, they experience a lower quality of life (19). However, in our study, energy and mental health were affected not only in physically inactive patients but also in physically active patients. The SF-36 mental health score was previously shown to be related to major depression in the elderly (20). Depressive symptoms were reported to be more prevalent compared to the pre-pandemic times (6). It has also been reported that both physical health and mental health of older people have been affected by the COVID-19 pandemic because of reduced physical activity, social isolation, and fear of COVID-19 infection (5).

The importance of physical activity in the management of pain has been highlighted in many studies (2), in which physical inactivity during the COVID-19 pandemic had a negative effect on pain severity among the elderly population. Similar to the findings of our study, but not in the elderly population, decreased physical activity during the pandemic was reported to be associated with pain augmentation in Japanese workers (9). Although pain severity in both male and female patients, as well as patients with and without sarcopenia, did not seem to be affected during the COVID-19 pandemic, the severity of pain was lower in women than in men and in patients with sarcopenia than in those without sarcopenia at both 2020 and 2021. Similar to our study, Zanin et al. reported a relationship between chronic pain and sarcopenia in 79 elderly women before the pandemic (21). Another study reported that women experience greater pain than men. Sex-based differences can be explained not only by hormonal differences and psychosocial factors, but also with sociodemographic factors (22).

Comparison of patients according to sex showed that not only pain severity but also SARC-F scores and quality of life in women was worse than in men in both 2020 and 2021. In addition, six of the eight quality-of-life parameters were affected in women, while three of them affected men during the COVID-19 pandemic. This result is consistent with that of previous studies, and poor quality of life and sarcopenia are generally higher in women around the world (23). These results can be explained by the lower physical activity levels of women, as observed in the female patients in our study. Furthermore, the socioeconomic and sociodemographic differences may have influenced the results. In studies investigating the impact of the pandemic on both males and females, Japanese investigators found that suicide deaths occurred more frequently in females since the onset of the pandemic (24). Another study reported that women had more musculoskeletal symptoms than men during the pandemic (25).

The main limitation of our study was evaluation of sarcopenia during follow-up without including objective measurements. The rating of musculoskeletal pain was done face to face interview in 2020, however one year later by telephone, they might not be equivalent, this was also a limitation of our study. In this pandemic, the Turkish elderly could not get outside for months, the questions asked in this study may not be sufficient to clearly reveal the troubles of this period, this is also another limitation of our study. However, to the best of our knowledge, our study is the first to evaluate the COVID-19 pandemic on the severity of pain, SARC-F, and quality of life in older patients. The strength of our cohort study was reporting the one-year follow-up results of patients according to having sarcopenia/not, sex, and being physically active/inactive. Further studies are needed to investigate the effects of this pandemic on sarcopenia using objective measurements.

In conclusion, the following findings were observed after one-year follow-up of elderly patients during the COVID-19 pandemic: 1) their quality of life decreased; 2) patients without sarcopenia were affected more than those with sarcopenia in terms of decrease in quality of life; 3) females were affect- 
ed more than males in terms of decrease in quality of life; 4) physically inactive patients were affected from this pandemic more than physically active patients in terms of not only decrease in quality of life, but also increase in the severity of pain; and 5) SARC-F scores did not change in any study group. This pandemic has and will continue to affect the quality of life and cause multiple lifestyle changes

\section{REFERENCES}

1. Ammar A, Brach M, Trabelsi K, et al. Effects of COVID-19 Home Confinement on Eating Behaviour and Physical Activity: Results of the ECLB-COVID19 International Online Survey. Nutrients 2020;12(6):1583. (PMID: 32481594)

2. Alzahrani H, Mackey M, Stamatakis E, et al. The association between physical activity and low back pain: A systematic review and meta-analysis of observational studies. Sci Rep 2019; 9:8244. (PMID: 31160632)

3. Vagetti GC, Barbosa Filho VC, Moreira NB, et al. Association between physical activity and quality of life in the elderly: a systematic review, 2000-2012. Braz. J. Psychiatry 2014; 36(1):76-88. (PMID: 24554274)

4. Kirwan R, McCullough D, Butler T, et al. Sarcopenia during COVID-19 lockdown restrictions: long-term health effects of short-term muscle loss. GeroScience 2020; 42(6):1547-78. (PMID: 33001410)

5. Bailey L, Ward M, DiCosimo A, et al. Physical and mental health of older people while cocooning during the COVID-19 pandemic. QJM-INT J MED 2021; hcab015. (PMID: 33471128)

6. Meiring RM, Gusso S, McCullough E, et al. The effect of the COVID-19 pandemic movement restrictions on self-reported physical activity and health in New Zealand: A cross-sectional survey. Int. J. Environ. Res. Public Health 2021; 18(4):1719. (PMID: 33578964)

7. Cruz-Jentoft AJ, Bahat G, Bauer J, et al. Sarcopenia: revised European consensus on definition and diagnosis. Age Ageing 2019; 48(1):16-31. (PMID: 30312372)

8. Hasegawa $Y$, Takahashi F, Hashimoto $Y$, et al. Effect of COVID-19 Pandemic on the Change in Skeletal Muscle Mass in Older Patients with Type 2 Diabetes: among older adults. Therefore, improving quality of life and increasing physical activities of older people are both economic and public health imperatives.

\section{Conflict of Interest}

All authors declare no conflict of interest.

A Retrospective Cohort Study. Int. J. Environ. Res. Public Health 2021; 18(8):4188. (PMID: 33920957)

9. Yoshimoto T, Fujii T, Oka H, et al. Pain Status and Its Association with Physical Activity, Psychological Stress, and Telework among Japanese Workers with Pain during the COVID-19 Pandemic. Int. J. Environ. Res. Public Health 2021; 18(11):5595. (PMID: 34073863)

10. Gallagher EJ, Liebman M, Bijur PE. Prospective validation of clinically important changes in pain severity measured on a visual analog scale. Ann Emerg Med 2001;38:633-8. (PMID: 11719741)

11. Kocyigit $H$, Aydemir $O$, Fisek $G$, et al. Validity and reliability of Turkish version of short form 36: a study of patients with rheumatoid disorder. J Drug Ther 1999;12:102-6. (in Turkish)

12. Bull FC, Al-Ansari SS, Biddle S, et al. World Health Organization 2020 guidelines on physical activity and sedentary behaviour. Br. J. Sports Med 2020;54:1451-62. (PMID: 33239350)

13. DiGiovanni G, Mousaw K, Lloyd T, et al. Development of a telehealth geriatric assessment model in response to the COVID-19 pandemic. Journal of geriatric oncology 2020;11(5):761-3. (PMID: 32327321)

14. Ida S, Kaneko R, Murata K. SARC-F for screening of sarcopenia among older adults: a meta-analysis of screening test accuracy. JAMDA 2018;19(8): 685-9. (PMID: 29778639)

15. Landi F, Cherubini A, Cesari M, et al. Sarcopenia and frailty: from theoretical approach into clinical practice. Eur Geriatr Med. 2016; 7(3), 197-200. (DOI:10.1016/j.eurger.2015.12.015)

16. Demirdel E, Demirdel S, Karahan S, et al. The cons of COVID-19 restrictions on physical activity in the el- 
derly; results of an online survey. Turkish J Geriatrics 2021; 24(1):32-40. (DOI: 10.31086/tjgeri.2021.197)

17. Aktürk Ü, Aktürk S, Erci B. The effects of depression, personal characteristics, and some habits on physical activity in the elderly. Perspect Psychiatr Care 2019;55(1):112-8. (PMID: 30171700)

18. Sekerci YG, Bicer EK. The Effect of Walking Exercise on Quality of Life and Sleep in Elderly Individuals: Randomized Controlled Study. Turkish J Geriatrics 2019; 22(4):443-53. (DOI:10.31086/tjgeri.2020.123)

19. Cunningham $C, O^{\prime}$ Sullivan $R$, Caserotti $P$, et al. Consequences of physical inactivity in older adults: A systematic review of reviews and meta-analyses. SCAND J MED SCI SPOR 2020; 30(5): 816-827. (PMID: 32020713)

20. Bruce F, Marnin H, Rachel D. Validity of the SF-36 five-item Mental Health Index for major depression in functionally impaired, community-dwelling elderly patients. J Am Geriatr Soc 2005;53(11):1978-85. (PMID: 16274382)

21. Zanin C, Candido JB, Jorge MSG, et al. Sarcopenia and chronic pain in institutionalized elderly wom- en. BrJP 2018;1(4):288-92. (DOI: 10.5935/25950118.20180055

22. Paller CJ, Campbell CM, Edwards RR, et al. Sexbased differences in pain perception and treatment. Pain medicine 2009;10(2):289-99. (PMID: 19207233)

23. Cherepanov D, Palta M, Fryback DG, et al. Gender differences in health-related quality-of-life are partly explained by sociodemographic and socioeconomic variation between adult men and women in the US: evidence from four US nationally representative data sets. Qual Life Res 2010;19(8):1115-24. (PMID: 20496168)

24. Nomura S, Kawashima T, Yoneoka D, et al. Trends in suicide in Japan by gender during the COVID-19 pandemic, up to September 2020. Psychiatry Res 2021; 295:113622. (PMID: 33290942)

25. Kirmizi M, Yalcinkaya G, Sengul YS. Gender differences in health anxiety and musculoskeletal symptoms during the COVID-19 pandemic. J Back Musculoskelet Rehabil 2021; 34(2):161-7. 1-7. (PMID: 33682695) 